\title{
Diagnostic efficiency of quantification of myocardial blood flow and coronary flow reserve with CZT dynamic SPECT imaging for patients with suspected coronary artery disease: a comparative study with traditional semi-quantitative evaluation
}

\author{
Jiao Wang ${ }^{1}$, Shuai Li ${ }^{1}$, Weiqiang Chen ${ }^{2}$, Yue Chen ${ }^{1}$, Zekun Pang ${ }^{1}$, Jianming Li $^{1}$ \\ ${ }^{1}$ Department of Nuclear Medicine, TEDA International Cardiovascular Hospital, Tianjin Medical University Clinical Cardiovascular Institute, \\ Tianjin, China; ${ }^{2}$ Department of Cardiology, TEDA International Cardiovascular Hospital, Tianjin Medical University Clinical Cardiovascular \\ Institute, Tianjin, China \\ Contributions: (I) Conception and design: All authors; (II) Administrative support: J Li; (III) Provision of study materials or patients: J Wang, Y Chen, \\ Z Pang; (IV) Collection and assembly of data: J Wang, S Li, W Chen; (V) Data analysis and interpretation: J Wang, J Li; (VI) Manuscript writing: \\ All authors; (VII) Final approval of manuscript: All authors. \\ Correspondence to: Jianming Li, PhD. Department of Nuclear Medicine, TEDA International Cardiovascular Hospital, Tianjin Medical University \\ Clinical Cardiovascular Institute, No.61, 3rd Road, Binhai New Area, Tianjin 300457, China. Email: ichlijm@163.com.
}

Background: Myocardial blood flow (MBF) quantitation with cadmium-zinc-telluride (CZT) dynamic single-photon emission computed tomography (SPECT) is being increasingly investigated toward clinical utilization.

Methods: In this prospective study, forty-nine patients with suspected or known coronary artery disease (CAD) underwent a rest/adenosine triphosphate (ATP) stress dynamic and routine gated myocardial perfusion imaging (MPI) by CZT SPECT and then received coronary angiography (CAG). Quantitative diagnosis from the dynamic SPECT and a flow diagram was automatically obtained by the dedicated software and compared with the result of semi-quantitative analysis with gated MPI using the angiographic stenosis as the reference standard.

Results: When stenosis $\geq 50 \%$ was considered at the participant level, the sensitivity (SN), specificity (SP), positive predictive value (PPV), negative predictive value (NPV) and accuracy (AC) of the quantitative diagnosis were higher than semi-quantitative method as $(84.4 \%$ vs. $65.6 \%, 88.2 \%$ vs. $70.6 \%, 93.1 \%$ vs. $80.8 \%, 75.0 \%$ vs. $52.2 \%, 85.7 \%$ vs. $67.3 \%$ ) (all $\mathrm{P}<0.05$ ). The receiver operating characteristic (ROC) curve analysis generated the optimal critical value as 1.86 and $1.61 \mathrm{~mL} / \mathrm{min} / \mathrm{g}$ for stress MBF (sMBF) and MFR, respectively. The diagnosis performance of the quantitative diagnosis was higher than semi-quantitative method as (78.9\% vs. $68.4 \%, 63.3 \%$ vs. $60.0 \%, 57.7 \%$ vs. $52.0 \%, 82.6 \%$ vs. $75.0 \%, 69.4 \%$ vs. $63.3 \%)$ for the criteria of $\geq 75 \%$ stenosis on CAG (all $\mathrm{P}<0.05$ ) with optimal critical values as 1.71 and $1.15 \mathrm{~mL} / \mathrm{min} / \mathrm{g}$. There was no significant difference between $\mathrm{SMBF}$ and MFR.

Conclusions: The diagnostic efficiency by using the quantitative method of CZT dynamic SPECT imaging is superior to traditional semi-quantitative gated MPI for the diagnosis of CAD, which improved the diagnostic specificity and accuracy when the critical was stenosis $\geq 50 \%$.

Keywords: Cadmium-zinc-telluride (CZT); single-photon emission computed tomography (SPECT); myocardial blood flow (MBF); coronary artery disease (CAD); diagnostic efficiency

Submitted Aug 20, 2020. Accepted for publication Dec 23, 2020.

doi: $10.21037 / \mathrm{cdt}-20-728$

View this article at: http://dx.doi.org/10.21037/cdt-20-728 


\section{Introduction}

Single-photon emission computed tomography (SPECT) myocardial perfusion imaging (MPI) plays an important role in the diagnosis, risk stratification and prognosis evaluation of coronary artery disease (CAD), with its clinical value being widely recognized $(1,2)$. Visual assessment and/or semi-quantitative analysis is often used in the diagnosis of CAD by traditional SPECT MPI, and the results are compared with the "normal reference segment" in the myocardium or "normal database" to judge whether the regional radioactivity distribution in the myocardium is abnormal and to determine the extent of CAD. Previous studies $(3,4)$ have shown that MPI may miss the diagnosis of left main lesions and/or balanced triple-vessel disease, while positron emission tomography computed tomography (PET/CT) quantitative myocardial blood flow (MBF) can significantly improve the diagnostic sensitivity of such conditions $(5,6)$. However, due to the limitations of equipment and radiopharmaceuticals, it still remains difficult for PET/CT quantitative MPI to be widely implemented in daily clinical practice until now, and PET/CT MPI is currently limited to a small number of heart centers.

In recent years, a new type of cardiac-dedicated SPECT, which uses a semi-conductor detector called cadmium zinc tellurium (CZT), has become more widely used in the clinic. It not only has high sensitivity and good spatial resolution, but also has a fast temporal resolution. It is capable of performing rapid and dynamic tomographic imaging, which makes it possible for the quantitative measurement of MBF (7). The advantage of this quantitative flow technique is that it can directly and sensitively detect left main, multivessel and/or balanced triple-vessel CAD, and avoid missing or underestimating the extent of disease. Coronary flow reserve (CFR), also known as myocardial flow reserve (MFR), is the ratio of stress to rest MBF, which is used to diagnose and evaluate the prognosis of CAD. The latest researches $(8,9)$ showed that there is good correlation between CZT SPECT and PET methods for quantitative measurement of MBF. There are however, limited reports on the diagnostic efficacy of using those quantitative blood flow parameters of CZT dynamic SPECT imaging for the diagnosis of CAD. Therefore, the purpose of our study is to explore the diagnostic efficacy and feasibility of using CZT dynamic SPECT imaging for the quantitative measurement of MBF and MFR in patients with suspected CAD in a single-center study, which is compared with traditional qualitative and semi-quantitative evaluation methods. We present the following article in accordance with the STRAD reporting checklist (available at http://dx.doi.org/10.21037/ cdt-20-728).

\section{Methods}

\section{Study population}

We studied 49 consecutive patients with suspected CAD. The inclusion criteria were as follows: Patients had to (I) be between 18 and 79 years old; (II) provide written informed consent; (III) had data of invasive coronary angiography (CAG) within 3 months before and after MPI examination, during which there was no revascularization treatment; (IV) be suitable for pharmacological stress MPI. Exclusion criteria: unstable angina pectoris; old myocardial infarction; post-revascularization; second-degree or higher atrioventricular block; sick sinus syndrome (except for those with pacemakers); chronic obstructive pulmonary disease (including asthma, bronchiectasis, emphysema, pulmonary fibrosis, etc.); severe hypotension (systolic blood pressure $<90 \mathrm{mmHg}$ ); severe mitral or aortic valve disease; cardiomyopathy (dilated, hypertrophic cardiomyopathy, etc.); those who fail to complete dynamic acquisition or complete dynamic acquisition and routine imaging, and/or whose image quality was not up to the requirements; female patients during pregnancy or lactation. All patients were all given informed consents.

\section{Imaging equipment and methods}

The imaging equipment used was equipped with a CZT detector with 19 pinhole collimators (NM530c, GE Healthcare, Milwaukee, WI, USA). The imaging agent used was ${ }^{99 \mathrm{~m}}$ Tc-methoxy isobutyl isonitrile (MIBI), which was provided by Beijing Senke Pharmaceutical Co., Ltd or Atomic Hi-Tech Tianjin Pharmaceutical Co., Ltd. Patients were required to not drink coffee, tea, or consume any foods containing caffeine or theophylline for 24 hours before the test, to stop taking routine drugs for cardiovascular diseases for 24 hours before the test, and to not smoke on the day of test.

Rest/stress dynamic SPECT imaging was carried out in a 1-day or 2-day protocol. Rest imaging was carried out in the supine position immediately after the patient drunk 350-500 $\mathrm{mL}$ water. An $18.5-37 \mathrm{MBq}$ dose of ${ }^{99 \mathrm{~m}} \mathrm{Tc}-\mathrm{MIBI}$ was preinjected to identify the patient's heart position, and after this pre-positioning, the dynamic acquisition program started for 10 seconds before a $185-296 \mathrm{MBq}$ dose of ${ }^{99 \mathrm{~m}} \mathrm{Tc}-\mathrm{MIBI}$ was 
injected (bolus injection, completed within 5 seconds), and data were continuously collected for 10 minutes in list mode. After dynamic acquisition, routine resting gated tomography was performed 40-60 minutes after the injection, with an acquisition time of 6 minutes. For patients following the 1-day protocol, stress imaging was performed after an interval of 1-4 hours, again, immediately after the patient drunk 350-500 mL water. After pre-positioning of the heart, adenosine triphosphate (ATP) stress was performed. The method of ATP stress performed was detailed in the study referenced (10). When the ATP stress was at its peak (3 min after starting the drug pumping), ${ }^{99 \mathrm{~m}} \mathrm{Tc}-\mathrm{MIBI}$ was injected (555-888 MBq, bolus injection, completed within 5 seconds) and data were continuously collected for 10 minutes in list mode. After dynamic acquisition, routine stress gated tomography was performed 40-60 minutes after injection, with an acquisition time of 4 minutes. For patients following the two-day protocol, the rest and stress doses were the same (370-555 MBq).The dynamic process for 2-day protocol was the same as 1-day protocol, and acquisition time of the routine rest and stress gated imaging was both 4 minutes. Routine gated acquisition parameters: 8 frames per cardiac cycle, $\pm 15 \%$ for heart rate window width, $140 \mathrm{keV}$ for energy peak, $\pm 10 \%$ for energy window width. All patients underwent a CT scan for obtaining attenuation correction map before SPECT imaging. The CT acquisition condition was as followings: $120 \mathrm{kV}, 50 \mathrm{~mA}$; ranging from the tip of the lung to the middle and lower part of the liver, and the equipment was GE NM690 (GE Healthcare, Milwaukee, WI, USA).

\section{Image processing and evaluation standards}

All raw data were transferred to Xeleris 3.0 (GE Healthcare, Milwaukee, WI, USA) and MyoFlowQ 1.0.2 (Beijing Larkcloud Biomedical, Beijing, China) workstations. The resting tomographic images, stress tomographic images and polar maps were iteratively reconstructed by the reconstruction module in Xeleris 3.0 and brought into the QPS (Cedars-Sinai Medical Center, LA, USA) program for further semiquantitative analysis. The dynamic list mode data were transferred to MyoFlowQ workstation and automatically re-binned into 18 frames consisting of $10 \times 10$ s, $5 \times 20 \mathrm{~s}, 2 \times 60 \mathrm{~s}, 1 \times 280 \mathrm{~s}$ frames. The CT attenuation correction data were then incorporated to complete CT and SPECT image fusion alignment, axis adjustment, attenuation and scattering correction. The regions of interest for input function and myocardial radioactivity sampling were automatically or manually set to obtain the dynamic curve and fitting curve of the left ventricular blood pool and left ventricular myocardium, and to calculate the rest MBF (rMBF) and stress MBF (sMBF) of the left ventricle $(\mathrm{LV})$, Unit: $\mathrm{mL} / \mathrm{min} / \mathrm{g}$. MFR was then obtained, calculated by the ratio of sMBF to rMBF. Resting systolic blood pressure multiplied by heart rate was used to correct rMBF. The rMBF, sMBF, and MFR of the LV were measured by MyoFlowQ software, and the quantitative diagnostic result was automatically judged by the software of MyoFlowQ, and the internal definition set by the software for positivity was as followings: myocardial ischemia was present with myocardial blood steal $\geq 3.01 \%$ of the whole myocardium, or when moderate abnormality was identified with myocardial ischemia and myocardial blood steal $\geq 20.3 \%$ of the whole myocardium. The above optimal cutoff values within the abnormal definition were calculated by ROC analysis which was approved by the product company(as mentioned above) (11). For semiquantitative diagnosis, the short-axis, horizontal long-axis, and vertical long-axis images of the $\mathrm{LV}$ were processed into the standard 17-segmental polar maps used by the American Heart Association (12). QPS was used to calculate the summed stress score (SSS), summed rest score (SRS), summed difference score (SDS), and transient ischemic dilatation (TID), SDS = SSS - SRS. The standard 5-point method (12) was used to judge the myocardial segmental radioactivity distribution: $0=$ normal; 1 = mild sparse; 2 = moderate sparse; $3=$ severe sparse; $4=$ defect. Semiquantitative diagnostic criteria $(13,14)$ were as followings: SSS $\geq 4$ or SDS $\geq 2$ or TID $\geq 1.19$ as CAD; SSS $<4$ and SDS $<2$ and TID $<1.19$ as none-CAD.

\section{The evaluation criteria of $C A G$}

CAG was performed using the standard Judkins method. Stenosis of coronary arteries with a diameter $\geq 2 \mathrm{~mm}$ was visually evaluated by two cardiologists with more than 3 years of interventional experience (consultation and settlement when there was a difference of opinion), and the main LV coronary artery and its main branches were assessed and the degree of stenosis determined and categorized into three situations: stenosis $<50 \%, 50 \% \leq$ stenosis $<75 \%$ and $75 \% \leq$ stenosis.

\section{Statistical analysis}

Using stenosis $\geq 50 \%$ and $\geq 75 \%$ on CAG as the reference 
criteria for the diagnosis of CAD, the diagnostic efficacy of semi-quantitative and quantitative methods of MPI were determined at the participant and vessel level, and included assessment of sensitivity ( $\mathrm{SN}$ ), specificity (SP), positive predictive value (PPV), negative predictive value (NPV) and accuracy (AC). A comparison of these methods was then carried out under different diagnostic criteria of CAG. Using stenosis $\geq 50 \%$ and $\geq 75 \%$ on CAG as the diagnostic reference criteria of CAD, rMBF, sMBF and LV-MFR of the $\mathrm{CAD}$ group and non-CAD group at the participant and vessel level were calculated and compared. Using stenosis $\geq 50 \%$ and $\geq 75 \%$ on CAG as the standard, ROC curves of sMBF and LV-MFR were made on the participant level, so the best critical values of sMBF and LV-MFR, and the corresponding diagnostic efficiency were respectively obtained and compared. Continuous data are expressed as mean \pm SD and categorical data as a percentage. All the data were processed by IBM SPSS 20.0, $t$-test, paired $t$-test, Wilcoxon sign rank and McNamara paired chi-square were used for statistical comparison according to the data type and its distribution. A value of $\mathrm{P}<0.05$ (two-sided) was considered statistically significant.

\section{Ethical statement}

The study was conducted in accordance with the Declaration of Helsinki (as revised in 2013). The study was approved by Ethics Institutional Board of Teda International Cardiovascular Hospital, China (No. 20180626-3) and informed consent was taken from all the patients.

\section{Results}

Forty-nine participants were enrolled with an average age of $62.5 \pm 8.2$ years, average height of $170 \pm 8 \mathrm{~cm}$, average weight of $73.3 \pm 11.6 \mathrm{~kg}$, and an average body mass index of $26.7 \pm 3.2 \mathrm{~kg} / \mathrm{m}^{2}$. The individual characteristics of the participants were shown in Table 1.

Using coronary artery stenosis reference standards of $\geq 50 \%$ and $\geq 75 \%$ on CAG, the diagnostic indexes of semiquantitative method, quantitative method, and combined method by MyoFlowQ were calculated and compared at the participant level $(n=49)$ under different reference criteria were compared, as shown in Table 2.

Table 2 showed that at the participant level, when CAG stenosis $\geq 50 \%$ was used as the reference standard, the $\mathrm{SN}$, SP, PPV and AC of quantitative diagnosis by the software
Table 1 Individual characteristics of participants $(n=49)$

\begin{tabular}{lc}
\hline Characteristic index & Value \\
\hline Gender (male) & $26(53.1 \%)$ \\
Age, years & $62.5 \pm 8.2$ \\
Body mass index $\left(\mathrm{kg} / \mathrm{m}^{2}\right)$ & $26.7 \pm 3.2$ \\
Typical angina pectoris & $30(61.2 \%)$ \\
Atypical angina pectoris & $19(38.8 \%)$ \\
Hypertension & $32(65.3 \%)$ \\
Hyperlipidemia & $19(38.8 \%)$ \\
Diabetes & $6(12.2 \%)$ \\
Smoking history & $14(28.6 \%)$ \\
Family history & $15(30.6 \%)$ \\
Negative (stenosis $<50 \%)$ on CAG & $16(32.7 \%)$ \\
Single vessel disease (stenosis $\geq 50 \%)$ & $13(26.5 \%)$ \\
Double vessel disease (stenosis $\geq 50 \%)$ & $8(16.3 \%)$ \\
Three-vessel disease (stenosis $\geq 50 \%)$ & $12(24.5 \%)$ \\
Mild lesion (stenosis $\geq 50 \%$, but $<75 \%)$ & $11(22.4 \%)$ \\
Moderate to severe lesions $($ stenosis $\geq 75 \%)$ & $22(44.9 \%)$ \\
\hline
\end{tabular}

were better than qualitative analysis $(\mathrm{P}<0.05)$, but there was no significant difference in NPV $(\mathrm{P}>0.05)$. The diagnostic efficiency of the combined method is significantly higher than that of the qualitative method, and the sensitivity of the combined method is higher than that of the quantitative method. When a higher CAG stenosis reference standard of $\geq 75 \%$ was used, the SN, NPV and AC of quantitative diagnosis were better than those of qualitative analysis $(\mathrm{P}<0.05)$, but there was no significant difference between $\mathrm{SP}$ and PPV $(\mathrm{P}>0.05)$. The SN, PPV, NPV and AC of combined method were significantly higher than qualitative method, and the SN, PPV, NPV and AC of combined method were higher than quantitative method. It could be seen that the diagnostic efficiency of combined method is higher than that of the single method. Typical cases were shown in Figures 1,2.

Coronary artery stenosis $<50 \%$ and $\geq 50 \%$ on CAG was used to separate negative (non-CAD, $\mathrm{n}=16$ ) and positive (CAD, n=33) groups. The differences of quantitative parameters at the participant and vessel level between the two groups were counted and compared, as shown in Table 3.

According to Table 3, using stenosis $\geq 50 \%$ on CAG as the reference standard, regardless of whether evaluation 
Table 2 Comparison between semi-quantitative method and quantitative method for diagnostic efficacy in MPI (participant level, n=49)

\begin{tabular}{|c|c|c|c|c|c|c|c|c|}
\hline $\begin{array}{l}\text { Diagnostic } \\
\text { indexes }\end{array}$ & \multicolumn{4}{|c|}{ Criteria 1: stenosis $\geq 50 \%$ on CAG } & \multicolumn{4}{|c|}{ Criteria 2 : stenosis $\geq 75 \%$ on CAG } \\
\hline SP & $70.6 \%$ & $88.2 \%$ & $75.0 \%$ & $<0.05^{\star}$ & $60.0 \%$ & $63.3 \%$ & $60.0 \%$ & 0.27 \\
\hline$A C$ & $67.3 \%$ & $85.7 \%$ & $83.7 \%$ & $<0.05^{\star}$ & $63.3 \%$ & $69.4 \%$ & $79.6 \%$ & $<0.05^{\star}$ \\
\hline
\end{tabular}

", combination method refers to the combination of qualitative and quantitative methods; ${ }^{*}$, the difference was statistically significant between qualitative method and quantitative method.

occurs at the participant level or vessel level, there was no significant difference in $\mathrm{LV}$-rMBF between the CAD group and non-CAD group $(\mathrm{P}>0.05)$, but the $L V$-sMBF and $L V$ $M F R$ in the CAD group were lower than in the non-CAD group $(\mathrm{P}<0.05)$.

Using stenosis $\geq 75 \%$ on CAG was used to separate negative (non-CAD, $n=29$ ) and positive $(C A D, n=20)$ groups. The differences of quantitative parameters at the participant and vessel level between the two groups were counted and compared, as shown in Table 4. The comparison results were the same as Table 3, that was, regardless of whether evaluation occurs at the participant level or vessel level, there was no significant difference in $\mathrm{LV}$-rMBF between the CAD group and the non-CAD group ( $\mathrm{P}>0.05)$, however the $\mathrm{LV}$-sMBF and LV-MFR in the $\mathrm{CAD}$ group were lower than in the non-CAD group $(\mathrm{P}<0.05)$.

Using stenosis $\geq 50 \%$ and $\geq 75 \%$ on CAG as reference standards respectively, the ROC curves of LV-sMBF, LVMFR, SSS and SDS in the diagnosis of CAD were made at the participant level, and the diagnostic efficacy of parameters were compared, as shown in Figures 3,4.

\section{Discussion}

The preliminary results of this study revealed that the diagnostic efficacy of using quantitative diagnostic method for CAD, was better than that of traditional semiquantitative method. Quantitative parameters from dynamic study with CZT SPECT, such as LV-sMBF and LVMFR, were lower in the CAD group than in the non-CAD group, but there was no significant difference in $\mathrm{rMBF}$. The ROC analysis showed that the diagnostic efficacy of either quantitative parameter of ischemia extent by a flow diagram or the single parameter (e.g., LV-sMBF or LVMFR) was higher than that of semi-quantitative parameter (e.g., SSS or SDS). And there was no significant difference in diagnostic value of LV-sMBF and LV-MFR. Previously, Chen et al. (11) found that SPECT MBF quantitation with a flow diagram appeared superior to a single MFR parameter in terms of the diagnostic performance. Part of the reasons can be attributed to that the method of flow diagram can provide an insight to reveal a small area of myocardial ischemia which was not yet large enough to reduce the MFR value to be marked as ischemia. And the parameter MFR can be highly affected by variation in rest MBF due to uncontrolled hypertension or pretest medications. In our study, we found that patient with large area of old myocardial infarction intended to overestimate MFR to $\geq 2.0$ because of ultralow rest MBF and slightly increased MBF in stress to preserve MFR in the infarcted area. Consequently, implementation of flow diagram by integrating complimentary information from rest MBF, stress MBF and MFR can be more robust to detect myocardial ischemia and possibly infarction than the single MFR parameter.

Past study (15) showed that the SN and SP of traditional SEPCT MPI in the diagnosis of suspected or diagnosed CAD were $86 \%$ and $74 \%$, respectively. With the clinical application of CZT SPECT, researches on the diagnostic efficacy of this equipment for CAD are emerging. A study on the diagnostic efficacy of CZT SPECT MPI for CAD (16) showed that even with stenosis $\geq 70 \%$ as the diagnostic criterion on CAG, the diagnostic SP of the device was only $69 \%$, but the SN could be as high as $95 \%$. The earliest study (17) on the efficacy of CZT SEPCT/ 

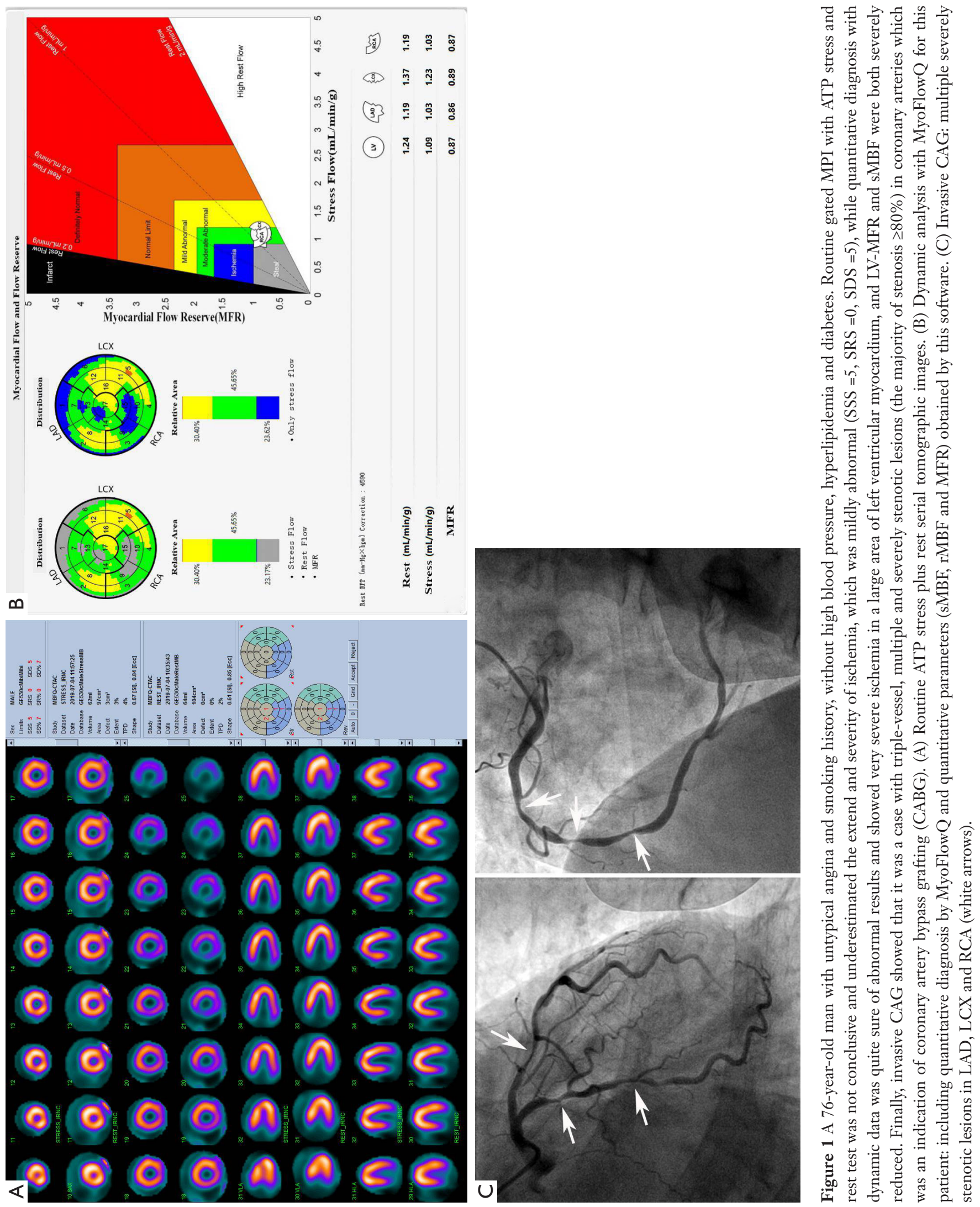

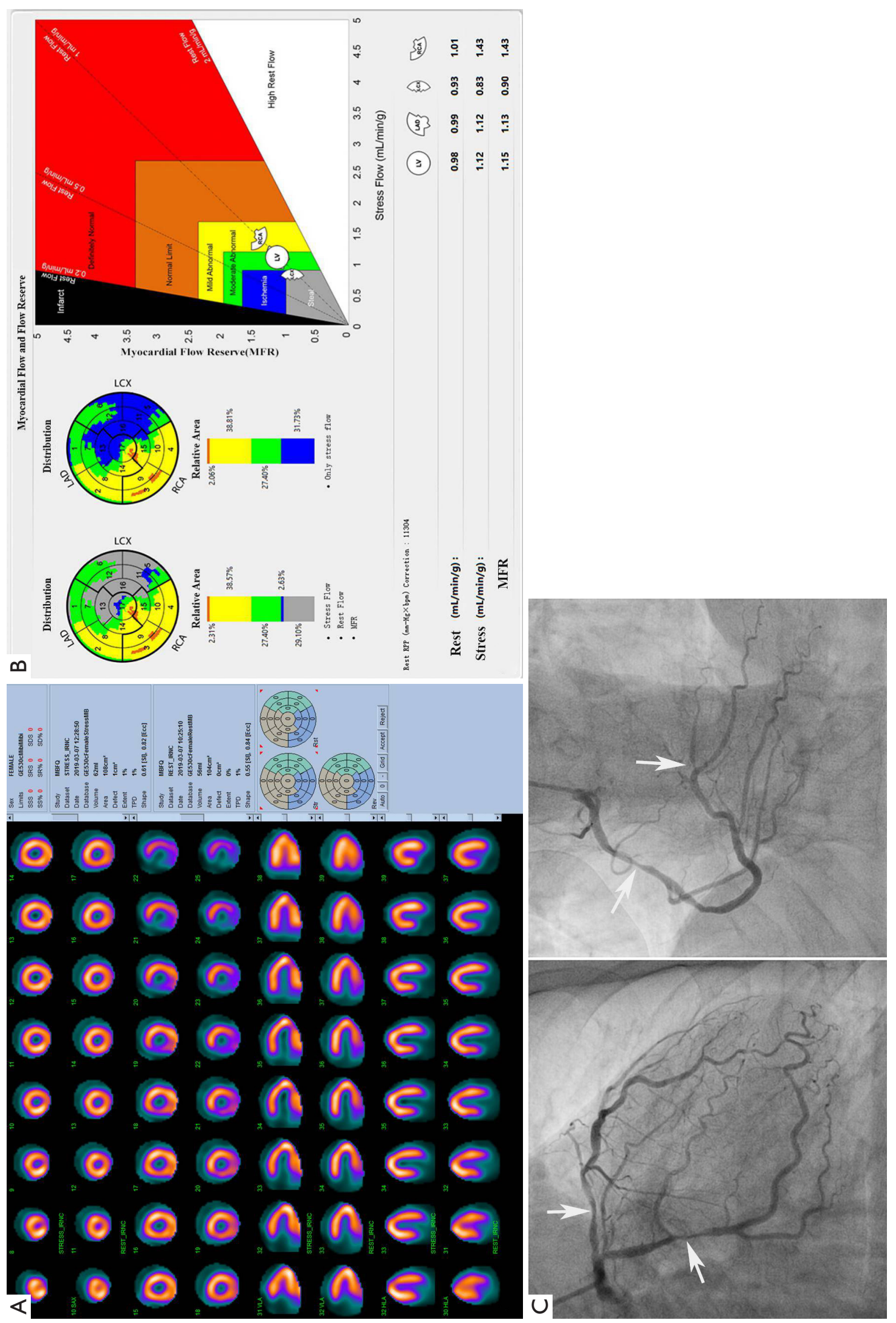

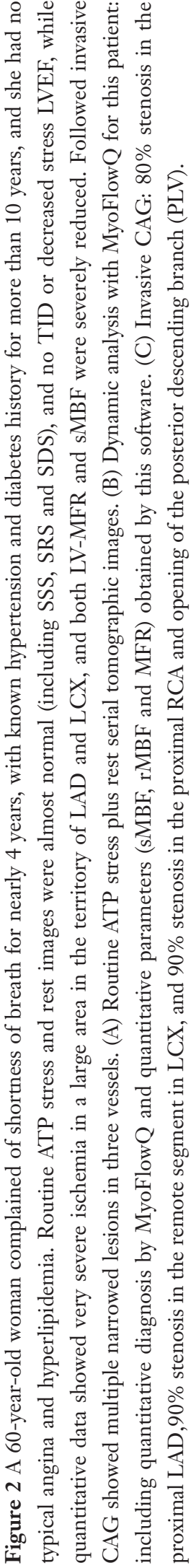


Table 3 Comparison of left ventricular quantitative parameters between the CAD group and non-CAD group (stenosis $\geq 50 \%$ on CAG as the case standard)

\begin{tabular}{|c|c|c|c|c|c|c|c|c|}
\hline $\begin{array}{l}\text { Quantitative } \\
\text { parameters }\end{array}$ & \multicolumn{4}{|c|}{ Participant level $(n=49)$} & \multicolumn{4}{|c|}{ Vessel level $(n=147)$} \\
\hline LV-sMBF $(\mathrm{mL} / \mathrm{min} / \mathrm{g})$ & $2.35 \pm 0.77$ & $1.41 \pm 0.41$ & 4.56 & $<0.05^{\star}$ & $2.05 \pm 0.80$ & $1.30 \pm 0.46$ & 7.08 & $<0.05^{\star}$ \\
\hline
\end{tabular}

*, the difference was statistically significant.

Table 4 Comparison of left ventricular quantitative parameters between the CAD group and the non-CAD group (stenosis $\geq 75 \%$ on CAG as the case standard)

\begin{tabular}{|c|c|c|c|c|c|c|c|c|}
\hline $\begin{array}{l}\text { Quantitative } \\
\text { parameters }\end{array}$ & \multicolumn{4}{|c|}{ Participant level $(n=49)$} & \multicolumn{4}{|c|}{ Vessel level $(n=147)$} \\
\hline LV-sMBF (mL/min/g) & $1.93 \pm 0.75$ & $1.32 \pm 0.42$ & 3.26 & $<0.05^{\star}$ & $2.07 \pm 0.85$ & $1.21 \pm 0.42$ & 7.23 & $<0.05^{*}$ \\
\hline
\end{tabular}

*, the difference was statistically significant.

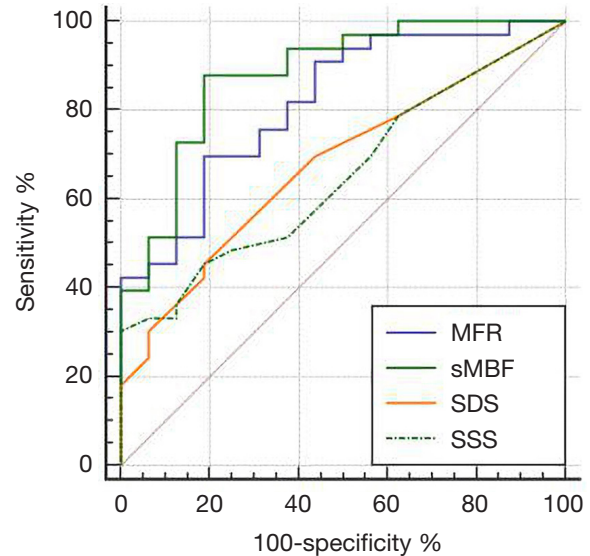

Figure 3 ROC curves of LV-sMBF, LV-MFR, SSS, SDS for the diagnosis of CAD (stenosis $\geq 50 \%$ as the case standard on CAG at participate level). The AUC of LV-sMBF, LV-MFR, SSS, SDS was $0.88,0.82,0.65,0.68$, respectively. The optimal critical value for $\mathrm{LV}$-sMBF was $1.86 \mathrm{~mL} / \mathrm{min} / \mathrm{g}$, and the SN and SP were $81.2 \%$ and $87.9 \%$, respectively. The optimal critical value for LV-MFR was 1.61 , and the SN and SP were $81.2 \%$ and $69.7 \%$, respectively. The optimal critical value for SSS was 8 , and the SN and SP were $30.3 \%$ and $100 \%$, respectively. The optimal critical value for SDS was 3, and the SN and SP were $45.5 \%$ and $81.2 \%$, respectively. There was no statistical significance for AUC between $\mathrm{LV}$-sMBF and $\mathrm{LV}$-CFR $(\mathrm{Z}=1.78, \mathrm{P}=0.08)$.



Figure 4 ROC curves of LV-sMBF, LV-MFR, SSS, SDS for the diagnosis of CAD (stenosis $\geq 75 \%$ as the case standard on CAG at participate level). The AUC of LV-sMBF, LV-MFR, SSS, SDS was $0.80,0.75,0.70,0.72$, respectively. The optimal critical value for $\mathrm{LV}$-sMBF was $1.71 \mathrm{~mL} / \mathrm{min} / \mathrm{g}$, and the SN and SP were $90.0 \%$ and $65.5 \%$, respectively. The optimal critical value for LV-MFR was 1.15 , and the $\mathrm{SN}$ and SP were $50.0 \%$ and $93.1 \%$, respectively. The optimal critical value for SSS was 5 , and the SN and SP were $60.0 \%$ and $79.3 \%$, respectively. The optimal critical value for SDS was 5 , and the $\mathrm{SN}$ and SP were $60.0 \%$ and $82 \%$, respectively. There was no statistical significance for AUC between $\mathrm{LV}$-sMBF and $\mathrm{LV}$-CFR $(\mathrm{Z}=1.34, \mathrm{P}=0.18)$. 
CT imaging in the diagnosis of CAD showed that when combined with CT attenuation correction, the $\mathrm{SN}, \mathrm{SP}$, and AC of diagnosis were $87 \%, 67 \%$, and $83 \%$, respectively. Thus, it can be seen that the SP of diagnosis of both traditional SPECT and CZT SPECT MPI is not ideal. A meta-analysis (18) has shown that although CZT SPECT improved the image quality and shortened acquisition time, the diagnostic SN of visual evaluation or semiquantitative analysis could reach 0.84 (95\% CI: 0.78-0.89), which was relatively satisfactory, however the diagnostic SP was only 0.69 (95\% CI: 0.62-0.76), which was similar as that of traditional SEPCT. Ito et al. (19) suggested that CZT SPECT combined with prone position and CT attenuation correction could be helpful in improving the accuracy of inferior wall and inferior lateral wall ischemia. Previous studies $(3,4)$ have suggested that the main reason for the poor specificity of SPECT MPI diagnosis was due to attenuation artifacts. Factors from image resolution, left main and/or balanced triple-vessel lesions would contribute to reduce the diagnostic sensitivity of SPECT MPI. These relevant issues were alleviated on PET/CT to give excellent diagnostic performance $(-90 \%)$ because of substantially higher image resolution and complete physical corrections (20). However, non-quantitative PET/CT MPI may still be hampered to identify balanced ischemia caused by left main and /or triple-vessel disease.

The emergence of quantitative MBF measurement technology provides a solution to these problems, which not only improves the diagnostic AC, SN and SP, but also has a very important role in evaluating the prognosis of patients (21). Previously, MBF quantitation has been mostly utilized in the field of PET/CT imaging and rarely used with SPECT or SPECT/CT imaging. Nonetheless, many practical limitations exist for the PET/CT approach, such as the high cost of PET/CT equipment, and it is also necessary to install an online cyclotron or buy a very expensive positron radionuclide generator to obtain positron perfusion imaging agents. Therefore, routine MPI and quantitative blood flow analysis with PET/CT have not been widely used in the clinics, particularly in Asian area. With the applicability of CZT cardiac-dedicated SPECT with not only higher sensitivity in photon detection but also the temporal resolution with list mode acquisition for continuous dynamic tomographic data acquisition, $\mathrm{MBF}$ quantitation with the SPECT technique become more promising toward routine clinical utilization. Recently, there have also been several studies utilizing SPECT cameras with rapid rotating gantry (RRG) for MBF quantitation. Ma et al. (22) compared and confirmed the similarity of RRG and CZT SPECT camera to quantify MBF. It was found that physical corrections along with other image corrections can provide comparable $\mathrm{MBF}$ quantitation in both congestive heart failure (CHF) and non-CHF patients, regardless of the type of SPECT systems used. The additional benefit of CZT SPECT over RGG-SPECT can be highlighted as reduced dose injection $(5-8 \mathrm{mCi})$ of perfusion tracer compared with the conventional dose of $>10 \mathrm{mCi}$ for RRG SPECT.

Because MPI quantitative blood flow diagnosis is not affected by soft-tissue attenuation artifacts (false positive, such as breast or diaphragm attenuation), left main artery and/or balanced triple-vessel stenosis (false negative or underestimating the extent and/or severity of ischemia), CZT SPECT quantitative blood flow imaging is expected as the solution. PET/CT is generally considered to be the best non-invasive method for quantitative blood flow analysis, so the accuracy of CZT SPECT quantitative measurement of $\mathrm{MBF}$ is the current focus of attention. An animal experimental study (23) showed that compared with radioactive microspheres (being widely regarded as the gold standard for experimental measuring MBF), ${ }^{201} \mathrm{Tl}$ or ${ }^{99 \mathrm{~m}} \mathrm{Tc}$ labeled perfusion imaging agent CZT SPECT (Discovery NM530c, GE Healthcare, Milwaukee, WI, USA) could be used to accurately measure MBF. A comparative study of ${ }^{99 \mathrm{~m}} \mathrm{Tc}$-Trofosmin CZT SPECT and ${ }^{13} \mathrm{~N}-\mathrm{NH}_{3}$ PET/CT (24) suggested that there was a good correlation between them, although the sMBF of CZT SPECT was relatively lower than that of PET/CT, which could lead to the underestimation in MFR to some extent.

The first head-to-head comparative analysis of ${ }^{99 \mathrm{~m}} \mathrm{Tc}$ MIBI CZT SPECT with ${ }^{15} \mathrm{O}$-water PET/CT and flow fraction reserve (FFR) (25) showed that blood flow reserve measured by PET/CT was similar to that measured by SPECT. Therefore, the application of CZT SPECT not only obtains the traditional MPI images and semiquantitative analysis, but also obtains the additional quantitative information of $\mathrm{rMBF}, \mathrm{sMBF}$ and MFR. Acampa et al. (26) used dynamic quantitative CZT SPECT in the diagnosis of obstructive $\mathrm{CAD}$, and found that $\mathrm{SMBF}$ and myocardial perfusion reserve (MPR) were lower in the CAD group than non-CAD group, with the similar result to our study. Univariate analysis showed that total perfusion defect (TPD), sMBF, and MPR were significant predictors for obstructive CAD, while multivariate analysis showed that MPR was an independent predictor of obstructive CAD. In one study using CZT SPECT quantitative imaging 
to diagnose 153 patients with suspected or diagnosed CAD (27), it was found that for triple-vessel disease, when the stress/resting MBF ratio was 1.3, diagnostic SN and SP of CZT SPECT was $93.3 \%$ and $75.9 \%$, respectively, significantly improving the detection rate of triple-vessel disease.

In a previous study (28) using ${ }^{201} \mathrm{Tl}$ CZT SPECT dynamic imaging to determine MPR to predict left main or triple-vessel disease, it was found that diagnosis achieved the highest efficiency when MPR $\leq 1.5$, with a $\mathrm{SN}$, SP, and AC of $86 \%, 78 \%$, and $80 \%$, respectively. The study concluded that dynamic quantitative determination of MPR by CZT SPECT could identify balanced ischemia in patients with left main or triple-vessel disease. Shiraishi et al. (29) assessed the feasibility of myocardial blood flow (MBF) and myocardial perfusion reserve (MPR) using dynamic SPECT with a CZT camera for estimating underlying CAD in patients with normal stress myocardial perfusion SPECT. They found that the MPR and MBF measured by CZT SPECT dynamic quantitative imaging were very useful in identifying balanced ischemia in patients with normal stress MPI. de Souza et al. (30) found that overall, MFR was lower in high-risk CAD patients than that in those with nonobstructive patients [1.99 (95\% CI: 1.22-2.84) vs. 2.89 (95\% CI: 2.22-3.58), $\mathrm{P}=0.026]$. In our study, patients with triplevessel disease accounted for $24.5 \%$ of participants (12/49), and the quantitative analysis of blood flow appeared to play a more important role in the diagnosis of these patients. It should also be noted that for patients with negative CAG but with coronary microvascular disease, MPI tomographic images may be normal, while sMBF and MFR could be significantly decreased. Under this circumstance, the positive results of quantitative blood flow would be defined as false positives if CAG was regarded as the reference standard.

\section{Study limitations}

This study has some limitations. First, the sample size is relatively small. Second, due to the limitation of technical accessibility, FFR and index of micro-vessel resistance (IMR), which reflect the function of epicardial coronary artery perfusion and micro-circulation, couldn't be compared as the gold standard.

\section{Conclusions}

The diagnostic efficiency by using the quantitative method of CZT dynamic SPECT imaging is superior to traditional semi-quantitative gated MPI for the diagnosis of CAD, which improved the diagnostic specificity and accuracy when the critical was stenosis $\geq 50 \%$.

\section{Acknowledgments}

The authors thank Dr. Bailing Hsu for his guidance of using the software of MyoFlowQ.

Funding: This work was supported by Key Project of Tianjin Health and Family Planning Commission, Tianjin City, China (grant number 16KG145) and Tianjin Binhai New Area Science and Technology Project (grant number 2018BWKZ007).

\section{Footnote}

Reporting Checklist: The authors have completed the STRAD reporting checklist. Available at http://dx.doi. org/10.21037/cdt-20-728

Data Sharing Statement: Available at http://dx.doi. org/10.21037/cdt-20-728

Conflicts of Interest: All authors have completed the ICMJE uniform disclosure form (available at http://dx.doi. org/10.21037/cdt-20-728). The authors have no conflicts of interest to declare.

Ethical Statement: The authors are accountable for all aspects of the work in ensuring that questions related to the accuracy or integrity of any part of the work are appropriately investigated and resolved. The study was conducted in accordance with the Declaration of Helsinki (as revised in 2013). The study was approved by Ethics Institutional Board of Teda International Cardiovascular Hospital, China (No. 2018-0626-3) and informed consent was taken from all the patients.

Open Access Statement: This is an Open Access article distributed in accordance with the Creative Commons Attribution-NonCommercial-NoDerivs 4.0 International License (CC BY-NC-ND 4.0), which permits the noncommercial replication and distribution of the article with the strict proviso that no changes or edits are made and the original work is properly cited (including links to both the formal publication through the relevant DOI and the license). See: https://creativecommons.org/licenses/by-nc- 
nd/4.0/.

\section{References}

1. Al Badarin FJ, Malhotra S. Diagnosis and Prognosis of Coronary Artery Disease with SPECT and PET. Curr Cardiol Rep 2019;21:57.

2. Taqueti VR, Di Carli, MF. Radionuclide myocardial perfusion imaging for the evaluation of patients with known or suspected coronary artery disease in the era of multimodality cardiovascular imaging. Prog Cardiovasc Dis 2015;57:644-53.

3. Berman DS, Kang X, Slomka PJ, et al. Underestimation of the extent of ischemia by gated SPECT myocardial perfusion imaging in patients with left main coronary artery disease. J Nucl Cardiol 2007;14:521-8.

4. Ghadri JR, Pazhenkottil AP, Nkoulou RN, et al. Very high coronary calcium score unmasks obstructive coronary artery disease in patients with normal SPECT MPI. Heart 2011;97:998-1003.

5. Ziadi MC. Myocardial flow reserve (MFR) with positron emission tomography (PET)/computed tomography (CT): clinical impact in diagnosis and prognosis. Cardiovasc Diagn Ther 2017;7:206-18.

6. Dorbala S, Di Carli MF. Cardiac PET perfusion: prognosis, risk stratification, and clinical management. Semin Nucl Med 2014;44:344-57.

7. Ben-Haim S, Murthy VL, Breault C, et al. Quantification of Myocardial Perfusion Reserve Using Dynamic SPECT Imaging in Humans: A Feasibility Study. J Nucl Med 2013;54:873-9.

8. Giubbini R, Bertoli M, Durmo R, et al. Comparison between N 13 NH 3-PET and 99m Tc-Tetrofosmin-CZT SPECT in the evaluation of absolute myocardial blood flow and flow reserve. J Nucl Cardiol 2019. [Epub ahead of print]. doi: 10.1007/s12350-019-01939-x.

9. Otaki Y, Manabe O, Miller Robert JH, et al. Quantification of myocardial blood flow by CZT-SPECT with motion correction and comparison with $\mathrm{O}$-water PET. J Nucl Cardiol 2019. [Epub ahead of print]. doi: 10.1007/s12350-019-01854-1.

10. Yao Zh, Zhu H, Li W, et al. Adenosine triphosphate stress myocardial perfusion imaging for risk stratification of patients aged 70 years and older with suspected coronary artery disease. J Nucl Cardiol 2017;24:429-33.

11. Chen LC, Hung HF, Jong BH, et al. A method to measure the extent of myocardial ischemia and steal with SPECT myocardial blood flow quantitation. Ann Nucl Med
2020;34:682-90.

12. Dorbala S, Ananthasubramaniam K, Armstrong IS, et al. Single Photon Emission Computed Tomography (SPECT) Myocardial Perfusion Imaging Guidelines: Instrumentation, Acquisition, Processing, and Interpretation. J Nucl Cardiol 2018;25:1784-846.

13. Kwon O, Hwang HJ, Koo HJ, et al. Ischemic burden assessment of myocardial perfusion CT, compared with SPECT using semi-quantitative and quantitative approaches. Int J Cardiol 2019;278:287-94.

14. Petretta M, Acampa W, Daniele S, et al. Transient ischemic dilation in SPECT myocardial perfusion imaging for prediction of severe coronary artery disease in diabetic patients. J Nucl Cardiol 2013;20:45-52.

15. Schuijf JD, Poldermans D, Shaw LJ, et al. Diagnostic and prognostic value of non-invasive imaging in known or suspected coronary artery disease. Eur J Nucl Med Mol Imaging 2006;33:93-104.

16. Duvall WL, Sweeny JM, Croft LB, et al. Comparison of high-efficiency CZT SPECT MPI to coronary angiography. J Nucl Cardiol 2011;18:595-604.

17. Fiechter M, Ghadri JR, Kuest SM, et al. Nuclear myocardial perfusion imaging with a novel cadmiumzinc-telluride detector SPECT/CT device: first validation versus invasive coronary angiography. Eur J Nucl Med Mol Imaging 2011;38:2025-30.

18. Nudi F, Iskandrian AE, Schillaci O, et al. Diagnostic Accuracy of Myocardial Perfusion Imaging With CZT Technology: Systemic Review and Meta-Analysis of Comparison With Invasive Coronary Angiography. JACC Cardiovasc Imaging 2017;10:787-94.

19. Ito S, Endo A, Okada T, et al. Comparison of CTAC and prone imaging for the detection of coronary artery disease using CZT SPECT. Ann Nucl Med 2017;31:629-35.

20. Di Carli MF, Dorbala S, Meserve J, et al. Clinical myocardial perfusion PET/CT. J Nucl Med 2007;48:783-93.

21. Murthy VL, Naya M, Forster CR, et al. Improved cardiac risk assessment with noninvasive measures of coronary flow reserve. Circulation 2011;124:2215-24.

22. Ma R, Wang L, Wu D, et al. Myocardial blood flow quantitation in patients with congestive heart failure: head-to-head comparison between rapid-rotating gantry SPECT and CZT SPECT. J Nucl Cardiol 2020;27:2287302.

23. Wells RG, Timmins R, Klein R, et al. Dynamic SPECT measurement of absolute myocardial blood flow in a porcine model. J Nucl Med 2014;55:1685-91. 
24. Nkoulou R, Fuchs TA, Pazhenkottil AP, et al. Absolute Myocardial Blood Flow and Flow Reserve Assessed by Gated SPECT with Cadmium-Zinc-Telluride Detectors Using 99mTc-Tetrofosmin: Head-to-Head Comparison with 13N-Ammonia PET. J Nucl Med 2016;57:1887-92.

25. Agostini D, Roule V, Nganoa C, et al. First validation of myocardial flow reserve assessed by dynamic Tc-sestamibi CZT-SPECT camera: head to head comparison with $\mathrm{O}$-water PET and fractional flow reserve in patients with suspected coronary artery disease. The WATER DAY study. Eur J Nucl Med Mol Imaging 2018;45:1079-90.

26. Acampa $W$, Assante R, Mannarino T, et al. Low-dose dynamic myocardial perfusion imaging by CZT-SPECT in the identification of obstructive coronary artery disease. Eur J Nucl Med Mol Imaging 2020;47:1705-12.

27. Miyagawa M, Nishiyama $Y$, Uetani T, et al. Estimation of myocardial flow reserve utilizing an ultrafast cardiac SPECT: Comparison with coronary angiography,

Cite this article as: Wang $\mathrm{J}, \mathrm{Li} \mathrm{S}$, Chen W, Chen Y, Pang Z, Li J. Diagnostic efficiency of quantification of myocardial blood flow and coronary flow reserve with CZT dynamic SPECT imaging for patients with suspected coronary artery disease: a comparative study with traditional semi-quantitative evaluation. Cardiovasc Diagn Ther 2021;11(1):56-67. doi: 10.21037/cdt20-728 fractional flow reserve, and the SYNTAX score. Int J Cardiol 2017;244:347-53.

28. Shiraishi S, Sakamoto F, Tsuda N, et al. Prediction of left main or 3-vessel disease using myocardial perfusion reserve on dynamic thallium-201 single-photon emission computed tomography with a semiconductor gamma camera. Circ J 2015;79:623-31.

29. Shiraishi S, Tsuda N, Sakamoto F, et al. Clinical usefulness of quantification of myocardial blood flow and flow reserve using CZT-SPECT for detecting coronary artery disease in patients with normal stress perfusion imaging. J Cardiol 2020;75:400-9.

30. de Souza ACDAH, Gonçalves BKD, Tedeschi AL, et al. Quantification of myocardial flow reserve using a gamma camera with solid-state cadmium-zinc-telluride detectors: Relation to angiographic coronary artery disease. J Nucl Cardiol 2019. [Epub ahead of print]. doi: 10.1007/s12350019-01775-z. 